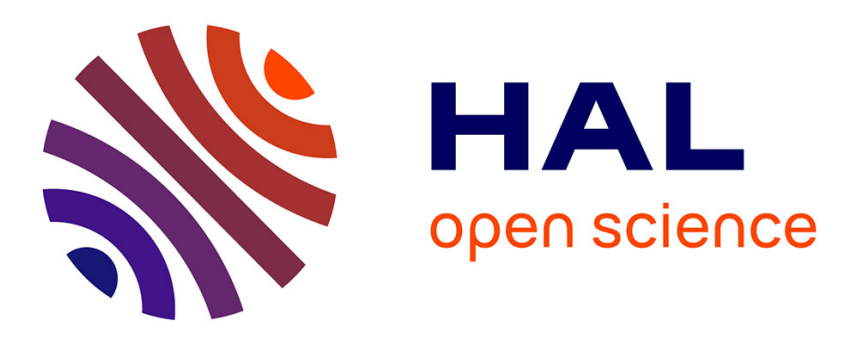

\title{
Homogeneity based Uniform Stability Analysis for Time-Varying Systems
}

\author{
Héctor Ríos, Denis Efimov, Leonid Fridman, Jaime Moreno, Wilfrid \\ Perruquetti
}

\section{- To cite this version:}

Héctor Ríos, Denis Efimov, Leonid Fridman, Jaime Moreno, Wilfrid Perruquetti. Homogeneity based Uniform Stability Analysis for Time-Varying Systems. IEEE Transactions on Automatic Control, 2016, 61 (3), pp.725-734. 10.1109/TAC.2015.2446371 • hal-01162678

\section{HAL Id: hal-01162678 \\ https://hal.inria.fr/hal-01162678}

Submitted on 11 Jun 2015

HAL is a multi-disciplinary open access archive for the deposit and dissemination of scientific research documents, whether they are published or not. The documents may come from teaching and research institutions in France or abroad, or from public or private research centers.
L'archive ouverte pluridisciplinaire HAL, est destinée au dépôt et à la diffusion de documents scientifiques de niveau recherche, publiés ou non, émanant des établissements d'enseignement et de recherche français ou étrangers, des laboratoires publics ou privés. 


\title{
Homogeneity based Uniform Stability Analysis for Time-Varying Systems
}

\author{
Héctor Ríos ${ }^{\dagger}$, Denis Efimov ${ }^{\dagger *}$, Leonid M. Fridman ${ }^{\ddagger}$, Jaime A. Moreno ${ }^{\S}$ and Wilfrid Perruquetti ${ }^{\dagger}$
}

\begin{abstract}
The uniform stability notion for a class of nonlinear time-varying systems is studied using the homogeneity framework. It is assumed that the system is weighted homogeneous considering the time variable as a constant parameter, then several conditions of uniform stability for such a class of systems are formulated. The results are applied to the problem of adaptive estimation for a linear system.
\end{abstract}

Index Terms-Time-varying systems, Stability of nonlinear systems, Adaptive control

\section{INTRODUCTION}

$\mathbf{T}$ HE global behavior of trajectories for homogeneous timeinvariant dynamical systems can be evaluated based on their behavior on a suitably defined sphere around the origin [1]. Thus, the local and global behaviors of homogeneous systems are the same. This property has been found useful for stability analysis (see, e.g. [1], [2], [3], [4] and [5]), approximation of system dynamics (see, e.g. [6] and [7]), highorder sliding-modes [8], stabilization (see, e.g. [9], [10], [11], [12] and [13]), and estimation (see, e.g. [2] and [7]). It has been shown that for stability/instability analysis, Lyapunov function of a homogeneous system can be chosen homogeneous (see, e.g. [5], [14] and [15]). Thus, the numerical analysis and design of homogeneous systems may be simpler since, for example, a Lyapunov function has to be only constructed on a sphere (on the whole state space it can extended using homogeneity). In addition, the homogeneous systems have certain intrinsic robustness properties (see, e.g. [16] and [17]).

In many cases the system dynamics is perturbed by exogenous disturbances, whose known parts can be modeled by some time functions, then another class of models arise: timevarying dynamical systems. Parameters of these disturbances (the rate of convergence or the main frequency) influence a lot on the system stability. For example, a nonlinear system

\footnotetext{
${ }^{\dagger}$ Non-A team @ Inria, Parc Scientifique de la Haute Borne, 40 avenue Halley, 59650 Villeneuve d'Ascq, France and CRIStAL (UMR CNRS 9189), Ecole Centrale de Lille, BP 48, Cité Scientifique, 59651 Villeneuve-d'Ascq, France. Emails: hector.rios_barajas@inria.fr; denis.efimoveinria.fr; wilfrid.perruquettieec-lille.fr

${ }^{\ddagger}$ Departamento de Control y Robótica, División de Ingeniería Eléctrica, Facultad de Ingeniería, Universidad Nacional Autónoma de México (UNAM), C.P. 04510, México, D.F., Mexico. Email: Ifridman@unam.mx

§Eléctrica y Computación, Instituto de Ingeniería, Universidad Nacional Autónoma de México (UNAM), C.P. 04510, México, D.F., Mexico. Email: JMorenoP@ii.unam.mx

* Department of Control Systems and Informatics, Saint Petersburg State University of Information Technologies Mechanics and Optics (ITMO), Kronverkskiy av. 49, Saint Petersburg, 197101, Russia.
}

can be stable for one exponentially converging disturbance and unstable with respect to another one, another example is the resonance phenomenon in linear systems. Moreover, in the adaptive control theory (see, e.g. [18] and [19]), the design of the adaptive law is crucial for the stability properties of the adaptive controller. Nevertheless, the adaptive law introduces a multiplicative nonlinearity that makes the closedloop plant nonlinear and time-varying. Because of this, the analysis and understanding of the stability and robustness of adaptive control schemes (nonlinear time-varying) are more challenging.

Due to robustness properties of homogeneous systems it would be interesting to apply this concept for time-varying systems. An extension of the homogeneity concept to timevarying systems has been given in [20] and [21], where in the latter a re-parametrization of time has also been required together with the state dilation. In this work, the weighted homogeneity theory is applied for the system dynamics considering the time variable as a constant parameter (it slightly differs from [21] and is similar to [20]).

Establishing stability properties, it is also important to quantify the rate of convergence in the system: exponential, asymptotic, finite-time or fixed-time (see, e.g. [12], [22], [23], [24] and [25]). Frequently, the homogeneity theory is used to establish finite-time or fixed-time stability (see, e.g. [2], [9], [25] and [26]): for example, if a system is globally asymptotically stable and homogeneous of negative degree, then it is finite-time stable. In this work we will also address the question of finite-time stability existence for time-varying systems.

The outline of this work is as follows. The preliminary definitions and the homogeneity framework are given in Section 2. The property of scaling of solutions for some class of homogeneous time-varying systems is presented in Section 3. The conditions of finite-time stability are given in Section 4. Application of the developed theory to the problem of stability and convergence analysis for an adaptive estimator is considered in Section 5.

\section{Preliminaries}

Consider a time-varying differential equation [27]:

$$
\frac{d x(t)}{d t}=f(t, x(t)), t \geq t_{0}, t_{0} \in \mathbb{R},
$$

where $x(t) \in \mathbb{R}^{n}$ is the state vector; $f: \mathbb{R}_{+} \times \mathbb{R}^{n} \rightarrow \mathbb{R}^{n}$ is a continuous function with respect to $x$ and measurable with 
respect to $t$, and $f(t, 0)=0$. It is assumed that solution of the system (1) for an initial condition $x_{0} \in \mathbb{R}^{n}$ at time instant $t_{0} \in \mathbb{R}$ is denoted as $x\left(t, t_{0}, x_{0}\right)$ and it is defined on some finite time interval $\left[t_{0}, t_{0}+T\right)$ where $0 \leq T<\infty$ (the notation $x(t)$ will be used to reference $x\left(t, t_{0}, x_{0}\right)$ if the origin of $x_{0}$ and $t_{0}$ is clear from the context).

A continuous function $\sigma: \mathbb{R}_{+} \rightarrow \mathbb{R}_{+}$belongs to class $\mathcal{K}$ if it is strictly increasing and $\sigma(0)=0$; it belongs to class $\mathcal{K}_{\infty}$ if it is also unbounded. A continuous function $\beta: \mathbb{R}_{+} \times \mathbb{R}_{+} \rightarrow \mathbb{R}_{+}$ belongs to class $\mathcal{K} \mathcal{L}$ if, for each fixed $s, \beta(r, s) \in \mathcal{K}$ with respect to $r$ and, for each fixed $r, \beta(r, s)$ is decreasing with respect to $s$ and $\beta(r, s) \rightarrow 0$ as $s \rightarrow \infty$. Denote a sequence of integers $1, \ldots, m$ as $\overline{1, m}$.

\section{A. Stability definitions}

Let $\Omega$ be an open subset of $\mathbb{R}^{n}$, such that $0 \in \Omega$.

Definition 1. [27] At the steady state $x=0$ the system (1) is said to be $e^{1}$

- US if for any $t_{0} \in \mathbb{R}$ and $\epsilon>0$ there is $\delta(\epsilon)$ such that for any $x_{0} \in \Omega$, if $\left|x_{0}\right| \leq \delta(\epsilon)$ then $\left|x\left(t, t_{0}, x_{0}\right)\right| \leq \epsilon$ for all $t \geq t_{0}$;

- UAS if it is US and for any $t_{0} \in \mathbb{R}, \kappa>0$ and $\epsilon>0$ there exists $T(\kappa, \epsilon) \geq 0$ such that for any $x_{0} \in \Omega$, if $\left|x_{0}\right| \leq \kappa$ then $\left|x\left(t, t_{0}, x_{0}\right)\right| \leq \epsilon$ for all $t \geq t_{0}+T(\kappa, \epsilon)$;

- UFTS if it is US and for any $x_{0} \in \Omega$ there exists $0 \leq T^{x_{0}}<+\infty$ such that $x\left(t, t_{0}, x_{0}\right)=0$ for all $t \geq t_{0}+T^{x_{0}} .^{2}$

If $\Omega=\mathbb{R}^{n}$, then $x=0$ is said to be globally $U S$ (GUS), UAS (GUAS), or UFTS (GUFTS), respectively. For US and UAS properties, there exist corresponding formulations in terms of functions from classes $\mathcal{K}_{\infty}$ and $\mathcal{K} \mathcal{L}$, respectively (see, e.g. [27]).

\section{B. Homogeneity}

For any $r_{i}>0, i=\overline{1, n}$ and $\lambda>0$, define the dilation matrix $\Lambda_{r}(\lambda)=\operatorname{diag}\left\{\lambda^{r_{i}}\right\}_{i=1}^{n}$ and the vector of weights $r=$ $\left[r_{1}, \ldots, r_{n}\right]^{T}$.

For any $r_{i}>0, i=\overline{1, n}$ and $x \in \mathbb{R}^{n}$ the homogeneous norm can be defined as follows:

$$
|x|_{r}=\left(\sum_{i=1}^{n}\left|x_{i}\right|^{\rho / r_{i}}\right)^{1 / \rho}, \rho=\prod_{i=1}^{n} r_{i} .
$$

For all $x \in \mathbb{R}^{n}$, its Euclidean norm $|x|$ is related with the homogeneous one:

$$
\underline{\sigma}_{r}\left(|x|_{r}\right) \leq|x| \leq \bar{\sigma}_{r}\left(|x|_{r}\right)
$$

for some $\underline{\sigma}_{r}, \bar{\sigma}_{r} \in \mathcal{K}_{\infty}$. In the following, due to this "equivalence", stability analysis with respect to the norm $|x|$ will be substituted with analysis for the norm $|x|_{r}$. The homogeneous norm has an important property that is $\left|\Lambda_{r}(\lambda) x\right|_{r}=\lambda|x|_{r}$ for all $x \in \mathbb{R}^{n}$. Define $\mathbb{S}_{r}=\left\{x \in \mathbb{R}^{n}:|x|_{r}=1\right\}$.

\footnotetext{
${ }^{1}$ The acronyms US, UAS and UFTS correspond to Uniformly Stable, Uniformly Asymptotically Stable and Uniformly Finite-Time Stable, respectively.

${ }^{2}$ The function $T_{0}\left(x_{0}\right)=\inf \left\{T^{x_{0}} \geq 0: x\left(t, t_{0}, x_{0}\right)=0 \forall t \geq t_{0}+T^{x_{0}}\right\}$ is called the uniform settling time of the system (1).
}

Definition 2. [1] The function $g: \mathbb{R}^{n} \rightarrow \mathbb{R}$ is called $r$ homogeneous $\left(r_{i}>0, i=\overline{1, n}\right)$, if for any $x \in \mathbb{R}^{n}$ the relation

$$
g\left(\Lambda_{r}(\lambda) x\right)=\lambda^{d} g(x)
$$

holds for some $d \in \mathbb{R}$ and all $\lambda>0$.

The function $f: \mathbb{R}^{n} \rightarrow \mathbb{R}^{n}$ is called $r$-homogeneous $\left(r_{i}>\right.$ $0, i=\overline{1, n}$ ), if for any $x \in \mathbb{R}^{n}$ the relation

$$
f\left(\Lambda_{r}(\lambda) x\right)=\lambda^{d} \Lambda_{r}(\lambda) f(x),
$$

holds for some $d \geq-\min _{1 \leq i \leq n} r_{i}$ and all $\lambda>0$. In both cases, the constant $d$ is called the degree of homogeneity.

A dynamical system

$$
\dot{x}(t)=f(x(t)), t \geq 0
$$

is called $r$-homogeneous of degree $d$ if this property is satisfied for the vector function $f$, in the sense of Definition 2. An advantage of homogeneous systems described by nonlinear ordinary differential equations is that any of its solution can be obtained from another solution under the dilation rescaling and a suitable time re-parametrization:

Proposition 1. [1] Let $x: \mathbb{R}_{+} \rightarrow \mathbb{R}^{n}$ be a solution of the $r$-homogeneous system (2) with the degree $d$ for an initial condition $x_{0} \in \mathbb{R}^{n}$. For any $\lambda>0$ define $y(t)=\Lambda_{r}(\lambda) x\left(\lambda^{d} t\right)$ for all $t \geq 0$, then $y(t)$ is also a solution of (2) with the initial condition $y_{0}=\Lambda_{r}(\lambda) x_{0}$.

In order to apply the weighted homogeneity property, introduced for time-invariant systems in Definition 2, to the timevarying systems (1), an extended concept is needed.

Definition 3. [20] The function $g: \mathbb{R}_{+} \times \mathbb{R}^{n} \rightarrow \mathbb{R}$ is called $r$-homogeneous $\left(r_{i}>0, i=\overline{1, n}\right)$, if for any $x \in \mathbb{R}^{n}$ and $t \in \mathbb{R}$ the relation

$$
g\left(t, \Lambda_{r}(\lambda) x\right)=\lambda^{d} g(t, x)
$$

holds for some $d \in \mathbb{R}$ and all $\lambda>0$.

The function $f: \mathbb{R}_{+} \times \mathbb{R}^{n} \rightarrow \mathbb{R}^{n}$ is called $r$-homogeneous $\left(r_{i}>0, i=\overline{1, n}\right)$, if for any $x \in \mathbb{R}^{n}$ and $t \in \mathbb{R}$ the relation

$$
f\left(t, \Lambda_{r}(\lambda) x\right)=\lambda^{d} \Lambda_{r}(\lambda) f(t, x)
$$

holds for some $d \geq-\min _{1 \leq i \leq n} r_{i}$ and all $\lambda>0$.

Thus in the time-varying case (1) the homogeneity will be verified considering $t$ as a constant parameter.

\section{MAIN RESULTS}

This section has three parts. First, an extension of Proposition 1 is presented for time-varying system (1), and some useful tools for uniform stability analysis of nonlinear timevarying systems are introduced. Second, relation with time scaling is analyzed. Third, the links with finite-time stability are investigated. 


\section{A. Scaling solutions of homogeneous time-varying systems}

Consider the following modification of the system (1), i.e.:

$$
\frac{d x(t)}{d t}=f(\omega t, x(t)), t \geq t_{0}, t_{0} \in \mathbb{R},
$$

for some $\omega>0$. The parameter $\omega$ represents dependence on the convergence rate of time processes in the system or the frequency of time-varying part. For an initial condition $x_{0} \in$ $\mathbb{R}^{n}$ at initial time $t_{0}$ denote the corresponding solution of (3) as $x_{\omega}\left(t, t_{0}, x_{0}\right)$, thus $x\left(t, t_{0}, x_{0}\right)=x_{1}\left(t, t_{0}, x_{0}\right)$, with $x\left(t, t_{0}, x_{0}\right)$ a solution of system (1). In this case the following extension of Proposition 1 (a variant of this result has been formulated in the proof of Theorem 2 in [20]) is given.

Proposition 2. Let $x\left(t, t_{0}, x_{0}\right)$ be a solution of the $r$ homogeneous system (1) with the degree $d$ for an initial condition $x_{0} \in \mathbb{R}^{n}$ and $t_{0} \in \mathbb{R}$. For any $\lambda>0$ with $\omega=\lambda^{d}$, the system (3) has a solution $y\left(t, t_{0}, y_{0}\right)=\Lambda_{r}(\lambda) x\left(\lambda^{d} t, \lambda^{d} t_{0}, x_{0}\right)$, for all $t \geq t_{0}$ with the initial condition $y_{0}=\Lambda_{r}(\lambda) x_{0}$.

Proof. Differentiating $y\left(t, t_{0}, y_{0}\right)$, it is obtained

$$
\begin{aligned}
\frac{d y\left(t, t_{0}, y_{0}\right)}{d t} & =\frac{d \Lambda_{r}(\lambda) x\left(\lambda^{d} t, \lambda^{d} t_{0}, x_{0}\right)}{d t} \\
& =\lambda^{d} \Lambda_{r}(\lambda) \frac{d x\left(\lambda^{d} t, \lambda^{d} t_{0}, x_{0}\right)}{d \lambda^{d} t}, \\
& =\lambda^{d} \Lambda_{r}(\lambda) f\left[\lambda^{d} t, x\left(\lambda^{d} t, \lambda^{d} t_{0}, x_{0}\right)\right], \\
& =f\left[\lambda^{d} t, \Lambda_{r}(\lambda) x\left(\lambda^{d} t, \lambda^{d} t_{0}, x_{0}\right)\right], \\
& =f\left[\lambda^{d} t, y\left(t, t_{0}, y_{0}\right)\right]
\end{aligned}
$$

and $y\left(t, t_{0}, y_{0}\right)$ is a solution of (3) for $\omega=\lambda^{d}$.

For the case $d=0$, it is recovered that all solutions of (1) are interrelated as in Proposition 1 (as in time-invariant case).

It is well known fact that for the ordinary differential equation (2) local attractiveness implies global asymptotic stability [1]. In the present setting that result has the following correspondence (a similar conclusion also can be found in the proof of Theorem 2 in [20]).

Lemma 1. Let the system (1) be r-homogeneous with degree $d \neq 0$ and GUAS at the origin, i.e. there is a function $\beta \in \mathcal{K} \mathcal{L}$ such that

$$
\left|x\left(t, t_{0}, x_{0}\right)\right|_{r} \leq \beta\left(\left|x_{0}\right|_{r}, t-t_{0}\right), \quad \forall t \geq t_{0},
$$

for any $x_{0} \in \mathbb{R}^{n}$ and any $t_{0} \in \mathbb{R}$. Then, the system (3) is GUAS at the origin for any $\omega>0$ and

$$
\left|x_{\omega}\left(t, t_{0}, x_{0}\right)\right|_{r} \leq \beta_{\omega}\left(\left|x_{0}\right|_{r}, t-t_{0}\right), \quad \forall t \geq t_{0},
$$

for any $x_{0} \in \mathbb{R}^{n}$ and any $t_{0} \in \mathbb{R}$, where $\beta_{\omega}(s, t)=$ $\omega^{1 / d} \beta\left(\omega^{-1 / d} s, \omega t\right)$.

Proof. Select $\omega>0$ and define $\lambda=\omega^{1 / d}$, which is well defined for any $\omega>0$ since $d \neq 0$. Define $y\left(t, t_{0}, y_{0}\right)=$ $\Lambda_{r}(\lambda) x\left(\lambda^{d} t, \lambda^{d} t_{0}, x_{0}\right)$ that, according to Proposition 2 , is a solution of (3) for the chosen $\omega$ and for all $t \geq t_{0}$ with the initial condition $y_{0}=\Lambda_{r}(\lambda) x_{0}$, then

$$
\begin{aligned}
\left|y\left(t, t_{0}, x_{0}\right)\right|_{r} & \leq \lambda\left|x\left(\lambda^{d} t, \lambda^{d} t_{0}, x_{0}\right)\right|_{r}, \\
& \leq \lambda \beta\left(\left|x_{0}\right|_{r}, \lambda^{d}\left[t-t_{0}\right]\right), \\
& \leq \lambda \beta\left(\lambda^{-1}\left|y_{0}\right|_{r}, \lambda^{d}\left[t-t_{0}\right]\right), \\
& =\omega^{1 / d} \beta\left(\omega^{-1 / d}\left|y_{0}\right|_{r}, \omega\left[t-t_{0}\right]\right),
\end{aligned}
$$

for all $t \geq t_{0}$ and for any $y_{0} \in \mathbb{R}^{n}$.

It is a well known fact that for linear time-varying systems (homogeneous systems of degree $d=0$ ) that its stability for some $\omega$ does not imply stability for all $\omega \in(0,+\infty)$. For nonlinear homogeneous time-varying systems with degree $d \neq$ 0 this is not the case, according to the result of Lemma 1 , if they are GUS for some $\omega$, then they preserve the uniform stability for an arbitrary $\omega>0$. This is an intriguing advantage of "nonlinear" time-varying systems. In addition, it is shown in Lemma 1 that the rate of convergence will be scaled by $\omega$, thus the time of transients in these systems is predefined by the time-varying part, which is not the case for the degree $d=0$, where the rate of convergence cannot be modified by $\omega$ (see Proposition 2)!

Further let us consider several useful consequences of Proposition 2 and Lemma 1.

Corollary 1. Let the system (1) be r-homogeneous with degree $d=0$ and UAS at the origin into the set $\Omega=B_{\rho}=\{x \in$ $\left.\mathbb{R}^{n}:|x|_{r} \leq \rho\right\}$, for some $0<\rho<+\infty$. Then, the system (1) is GUAS at the origin.

Proof. In this case there is a function $\beta \in \mathcal{K} \mathcal{L}$ such that

$$
\left|x\left(t, t_{0}, x_{0}\right)\right|_{r} \leq \beta\left(\left|x_{0}\right|_{r}, t-t_{0}\right), \quad \forall t \geq t_{0},
$$

for any $x_{0} \in B_{\rho}$ and any $t_{0} \in \mathbb{R}$. Take any $\bar{x}_{0} \notin B_{\rho}$, then there is $x_{0} \in B_{\rho}$, with $\left|x_{0}\right|_{r}=\rho$, such that $\bar{x}_{0}=\Lambda_{r}(\lambda) x_{0}$, with $\lambda=$ $\left|\bar{x}_{0}\right|_{r} \rho^{-1}$. By Proposition 2, $x\left(t, t_{0}, \bar{x}_{0}\right)=\Lambda_{r}(\lambda) x\left(t, t_{0}, x_{0}\right)$ is the corresponding solution of (1) and

$$
\begin{aligned}
\left|x\left(t, t_{0}, \bar{x}_{0}\right)\right|_{r} & =\lambda\left|x\left(t, t_{0}, x_{0}\right)\right|_{r}, \\
& \leq \lambda \beta\left(\left|x_{0}\right|_{r}, t-t_{0}\right), \\
& =\left|\bar{x}_{0}\right|_{r} \rho^{-1} \beta_{\omega}\left(\rho, t-t_{0}\right),
\end{aligned}
$$

for all $t \geq t_{0}$.

Corollary 2. Let the system (3) be r-homogeneous with degree $d \neq 0$ and UAS at the origin into the set $\Omega=B_{\rho}=\{x \in$ $\left.\mathbb{R}^{n}:|x|_{r} \leq \rho\right\}$, for a fixed $0<\rho<+\infty$, for any $\omega>0$. Then, the system (3) is GUAS at the origin, for any $\omega>0$.

Proof. In this case, for any $\omega>0$, there is some function $\beta_{\omega} \in \mathcal{K} \mathcal{L}$ such that

$$
\left|x_{\omega}\left(t, t_{0}, x_{0}\right)\right|_{r} \leq \beta_{\omega}\left(\left|x_{0}\right|_{r}, t-t_{0}\right), \quad \forall t \geq t_{0},
$$

for any $x_{0} \in B_{\rho}$ and any $t_{0} \in \mathbb{R}$, the functions $\beta_{\omega}$ have a continuous dependence on $\omega$ since the solutions of (3) depend continuously on the parameter $\omega$ [27]. Note that, for any $\bar{x}_{0} \notin$ $B_{\rho}$ there is $x_{0} \in B_{\rho}$, with $\left|x_{0}\right|_{r}=\rho$, such that $\bar{x}_{0}=\Lambda_{r}(\lambda) x_{0}$, 
with $\lambda=\left|\bar{x}_{0}\right|_{r} \rho^{-1}$. Select $\omega=\lambda^{-d}$, then, $x_{1}\left(t, t_{0}, \bar{x}_{0}\right)=$ $\Lambda_{r}(\lambda) x_{\omega}\left(\lambda^{d} t, \lambda^{d} t_{0}, x_{0}\right)$, by Proposition 2 , and

$$
\begin{aligned}
\left|x_{1}\left(t, t_{0}, \bar{x}_{0}\right)\right|_{r} & =\lambda\left|x_{\omega}\left(\lambda^{d} t, \lambda^{d} t_{0}, x_{0}\right)\right|_{r}, \\
& \leq \lambda \beta_{\omega}\left(\left|x_{0}\right|_{r}, \lambda^{d}\left[t-t_{0}\right]\right), \\
& =\left|\bar{x}_{0}\right|_{r} \rho^{-1} \beta_{\omega}\left(\rho,\left|\bar{x}_{0}\right|_{r}^{d} \rho^{-d}\left[t-t_{0}\right]\right),
\end{aligned}
$$

for all $t \geq t_{0}$. Therefore, $\left|x_{1}\left(t, t_{0}, \bar{x}_{0}\right)\right|_{r} \leq \rho^{-1} \beta_{\omega}(\rho, 0)\left|\bar{x}_{0}\right|_{r}$ for all $t \geq t_{0}$ and all $\bar{x}_{0} \in \mathbb{R}^{n}$. Take $\delta>\rho$, then $\omega \in\left[\delta^{-d} \rho^{d}, 1\right]$ for $\rho \leq\left|\bar{x}_{0}\right|_{r} \leq \delta$, and there is $p_{\delta, \rho}=\sup _{\omega \in\left[\delta^{-d} \rho^{d}, 1\right]} \beta_{\omega}(\rho, 0)$, then $\left|x_{1}\left(t, t_{0}, \bar{x}_{0}\right)\right|_{r} \leq \max \left\{\rho^{-1} p_{\delta, \rho}\left|\bar{x}_{0}\right|_{r}, \beta_{1}\left(\left|\bar{x}_{0}\right|_{r}, 0\right)\right\}$ for all $t \geq t_{0}$ and all $\left|\bar{x}_{0}\right|_{r} \leq \delta$, and the system (3), for $\omega=1$, is GUS. In addition, for any $\kappa>0$ and $\epsilon>0$, if $\rho \leq\left|\bar{x}_{0}\right|_{r} \leq \kappa$, then

$$
\left|x_{1}\left(t, t_{0}, \bar{x}_{0}\right)\right|_{r} \leq \kappa \rho^{-1} \beta_{\omega}\left(\rho, t-t_{0}\right),
$$

and by the properties of the functions from class $\mathcal{K} \mathcal{L}$, there is $\hat{T}(\kappa, \epsilon) \geq 0$ such that $\kappa \rho^{-1} \beta_{\omega}[\rho, \hat{T}(\kappa, \epsilon)] \leq \epsilon$, for any $\omega \in\left[\kappa^{-d} \rho^{d}, 1\right]$. Consequently, if $\left|\bar{x}_{0}\right|_{r} \leq \kappa$, then for any $\epsilon>0,\left|x_{1}\left(t, t_{0}, \bar{x}_{0}\right)\right|_{r} \leq \epsilon$ for $t \geq t_{0}+\max \{\hat{T}(\kappa, \epsilon), \hat{T}(\kappa, \rho)+$ $\left.T_{1}(\rho, \epsilon)\right\}$, where $T_{1}(\kappa, \epsilon)$ is the solution of the equation $\beta_{1}\left[\kappa, T_{1}(\kappa, \epsilon)\right]=\epsilon$, and therefore, the system (3), for $\omega=1$, is GUAS. The claim for all $\omega>0$ follows from Lemma 1.

Corollary 3. Let the system (3) be r-homogeneous with degree $d>0$ and UAS at the origin into the set $B_{\rho}$ for some $0<$ $\rho<+\infty$, for any $0 \leq \omega \leq \omega_{0}$, with $0<\omega_{0}<+\infty$. Then, the system (3) is GUAS at the origin, for any $\omega>0$.

Proof. In this case, again for any $\omega>0$, there is some function $\beta_{\omega} \in \mathcal{K} \mathcal{L}$ such that

$$
\left|x_{\omega}\left(t, t_{0}, x_{0}\right)\right|_{r} \leq \beta_{\omega}\left(\left|x_{0}\right|_{r}, t-t_{0}\right), \quad \forall t \geq t_{0},
$$

for any $x_{0} \in B_{\rho}$ and any $t_{0} \in \mathbb{R}$, the functions $\beta_{\omega}$ have a continuous dependence on $\omega$. Take $\bar{x}_{0} \notin B_{\rho}$ and select $1<\lambda=\rho^{-1}\left|\bar{x}_{0}\right|_{r}$, then $\bar{x}_{0}=\Lambda_{r}(\lambda) x_{0}$, for some $x_{0} \in B_{\rho}$, with $\left\|x_{0}\right\|_{r}=\rho$. Further, according to Proposition 2, $x_{\omega_{0}}\left(t, t_{0}, \bar{x}_{0}\right)=\Lambda_{r}(\lambda) x_{\omega}\left(\lambda^{d} t, \lambda^{d} t_{0}, x_{0}\right)$ for all $t \geq t_{0}$, where $\omega=\lambda^{-d} \omega_{0} \leq \omega_{0}$ since $d>0$ and $\lambda>1$, and

$$
\begin{aligned}
\left|x_{\omega_{0}}\left(t, t_{0}, x_{0}\right)\right|_{r} & \leq \lambda\left|x_{\omega}\left(\lambda^{d} t, \lambda^{d} t_{0}, x_{0}\right)\right|_{r}, \\
& \leq \lambda \beta_{\omega}\left(\left|x_{0}\right|_{r}, \lambda^{d}\left[t-t_{0}\right]\right), \\
& =\rho^{-1}\left|\bar{x}_{0}\right|_{r} \beta_{\omega}\left(\rho, \rho^{-d}\left|\bar{x}_{0}\right|_{r}^{d}\left[t-t_{0}\right]\right) .
\end{aligned}
$$

Next, applying similar arguments as in the proof of Corollary 2 , the global uniform asymptotic stability at the origin of the system (3) for $\omega_{0}$ can be substantiated. Using the result of Lemma 1 the claim about global uniform asymptotic stability with respect to all $\omega>0$ can be recovered.

Corollary 4. Let the system (3) be r-homogeneous with degree $d<0$ and UAS with respect to the set $B_{\rho}$ for some $0<\rho<$ $+\infty$, for any $0 \leq \omega \leq \omega_{0}$, with $0<\omega_{0}<+\infty{ }^{3}$. Then, the system (3) is GUAS at the origin, for any $\omega>0$.

Proof. Take $\rho^{\prime}>\rho$ and $\lambda=\frac{\left|\bar{x}_{0}\right|_{r}}{\rho^{\prime}} \leq 1$ for any $\bar{x}_{0} \in$ $B_{\rho}$, then there exists $x_{0} \in B_{\rho^{\prime}},\left|x_{0}\right|_{r}=\rho^{\prime}$ such that

${ }^{3}$ In this case, for each $0 \leq \omega \leq \omega_{0}$, any $\varepsilon>0$ and $\kappa \geq 0$, there is $0 \leq T_{\kappa, \varepsilon}^{\omega}<+\infty$, such that $\left|x_{\omega}\left(t, t_{0}, x_{0}\right)\right|_{r} \leq \rho+\varepsilon$, for all $t \geq t_{0}+T_{\kappa, \varepsilon}^{\omega}$, for any $x_{0} \in B_{\kappa}$, and $\left|x\left(t, t_{0}, x_{0}\right)\right|_{r} \leq \sigma_{\omega}\left(\left|x_{0}\right|_{r}\right)$, for all $t \geq t_{0}$, for some function $\sigma_{\omega} \in \mathcal{K}$, for all $t_{0} \in \mathbb{R}$.
$\bar{x}_{0}=\Lambda_{r}(\lambda) x_{0}$. Define $\omega=\lambda^{-d} \omega_{0}$, then $\omega \leq \omega_{0}$, for $\lambda<1$ and $d<0$. According to Proposition 2, $x_{\omega_{0}}\left(t, t_{0}, \bar{x}_{0}\right)=\Lambda_{r}(\lambda) x_{\omega}\left(\lambda^{d} t, \lambda^{d} t_{0}, x_{0}\right)$, for all $t \geq t_{0}$. Since $\left|x_{\omega}\left(t, t_{0}, x_{0}\right)\right|_{r} \leq \sigma_{\omega}\left(\left|x_{0}\right|_{r}\right)$, for all $t \geq t_{0}$, then $\left|x_{\omega_{0}}\left(t, t_{0}, \bar{x}_{0}\right)\right|_{r} \leq \lambda \sigma_{\omega}\left(\lambda^{-1}\left|\bar{x}_{0}\right|_{r}\right)=\rho^{\prime-1}\left|\bar{x}_{0}\right|_{r} \sigma_{\omega}\left(\rho^{\prime}\right)$, for all $t \geq t_{0}$ and all $\bar{x}_{0} \in B_{\rho}$. Define $R=\sup _{\omega \in\left[0, \omega_{0}\right]} \sigma_{\omega}\left(\rho^{\prime}\right)$, then $\left|x_{\omega_{0}}\left(t, t_{0}, \bar{x}_{0}\right)\right|_{r} \leq \eta\left(\left|\bar{x}_{0}\right|_{r}\right)=\max \left\{\rho^{\prime-1}\left|\bar{x}_{0}\right|_{r} R, \sigma_{\omega_{0}}\left(\left|\bar{x}_{0}\right|_{r}\right)\right\}$, for all $t \geq t_{0}$, for any $\bar{x}_{0} \in \mathbb{R}^{n}$, and the system (3) is GUS for $\omega_{0}$. Select $\varepsilon>0$ and take $\lambda=\frac{\eta^{-1}(\varepsilon)}{\rho+\varepsilon}<1$, then, for any $\bar{x}_{0} \in B_{\rho} \backslash B_{\eta^{-1}(\varepsilon)}$, there exists $x_{0} \notin B_{\rho}$, with $\left|x_{0}\right|_{r} \leq \lambda^{-1} \rho$, such that $\bar{x}_{0}=\Lambda_{r}(\lambda) x_{0}$. Since again $x_{\omega_{0}}\left(t, t_{0}, \bar{x}_{0}\right)=\Lambda_{r}(\lambda) x_{\omega}\left(\lambda^{d} t, \lambda^{d} t_{0}, x_{0}\right)$, for all $t \geq t_{0}$, and $\left|x_{\omega}\left(\lambda^{d} t, \lambda^{d} t_{0}, x_{0}\right)\right|_{r} \leq \rho+\varepsilon$, for all $\lambda^{d} t \geq \lambda^{d} t_{0}+T_{\lambda^{-1} \rho, \varepsilon}^{\omega}$, then $\left|x_{\omega_{0}}\left(t, t_{0}, \bar{x}_{0}\right)\right|_{r} \leq \eta^{-1}(\varepsilon) \leq \varepsilon$, for all $t \geq t_{0}+\lambda^{-d} T_{\lambda^{-1} \rho, \varepsilon}^{\rho, \varepsilon}$. For $\bar{x}_{0} \in B_{\eta^{-1}(\varepsilon)}$, it is obtained that $\left|x_{\omega_{0}}\left(t, t_{0}, \bar{x}_{0}\right)\right|_{r} \leq \varepsilon$, for all $t \geq t_{0}$, that implies uniform attractivity of the origin for system (3) for $\omega_{0}$. From these properties the global uniform asymptotic stability for $\omega_{0}$ is deduced. Finally, the claim of the corollary for any $\omega \in(0,+\infty)$ follows from the conclusion of Lemma 1.

From this proof it is given that the requirements for $\omega=0$ can be skipped if the system is stable, and thus, the following corollary arrives.

Corollary 5. Let the system (3) be r-homogeneous with degree $d<0$ and uniformly asymptotically stable with respect to the set $B_{\rho}$ for some $0<\rho<+\infty$, for any $0<\omega \leq \omega_{0}$, with $0<\omega_{0}<+\infty^{4}$. If the system (3) is US, for any $\omega>0$, then it is GUAS at the origin, for any $\omega>0$.

The results presented in this section open a possibility for stability analysis of nonlinear time-varying systems. In particular, they can be interpreted as robustness of the timeinvariant homogeneous systems with respect to time-varying perturbations of parameters, i.e. if for one value of parameter $\omega$ for this time-varying perturbation the system is stable, then it can be stable for any other value of $\omega$.

\section{B. Robustness with respect to time scaling}

Consider, for some $a>0$, the following modification of (1) or (3)

$$
\frac{d z(t)}{d t}=a f(a t, z(t)), t \geq t_{0}, t_{0} \in \mathbb{R}
$$

where $z \in \mathbb{R}^{n}$ is the state. Then, $z\left(t, t_{0}, x_{0}\right)=x\left(a t, a t_{0}, x_{0}\right)$, for $t \geq t_{0}$, is a solution of system (4), for the initial condition $x_{0} \in \mathbb{R}^{n}$ and $x\left(t, t_{0}, x_{0}\right)$ a solution of system (1). Indeed,

$$
\begin{aligned}
\frac{d z\left(t, t_{0}, x_{0}\right)}{d t} & =\frac{d x\left(a t, a t_{0}, x_{0}\right)}{d t}, \\
& =a \frac{d x\left(a t, a t_{0}, x_{0}\right)}{d a t}, \\
& =a f\left[a t, x\left(a t, a t_{0}, x_{0}\right)\right], \\
& =a f\left(a t, z\left(t, t_{0}, x_{0}\right)\right) .
\end{aligned}
$$

${ }^{4}$ For each $0<\omega \leq \omega_{0}$, any $\varepsilon>0$ and $\kappa \geq 0$, there is $0 \leq T_{\kappa, \varepsilon}^{\omega}<+\infty$, such that $\left|x_{\omega}\left(t, t_{0}, x_{0}\right)\right|_{r} \leq \rho+\varepsilon$, for all $t \geq t_{0}+T_{\kappa, \varepsilon}^{\omega}$, for any $x_{0} \in B_{\kappa}$, and $\left|x\left(t, t_{0}, x_{0}\right)\right|_{r} \leq \sigma_{\omega}\left(\left|x_{0}\right|_{r}\right)$, for all $t \geq t_{0}$, for some function $\sigma_{\omega} \in \mathcal{K}$, for all $t_{0} \in \mathbb{R}$. 
Thus time scaling (multiplication on $a$ ) acts similarly on solutions as the dilation transformation in homogeneous systems, and the following conclusion on the system stability can be obtained.

Lemma 2. Let the system (1) be GUAS at the origin. Then, the system (4) is GUAS at the origin, for any $a>0$, and the rate of convergence in (4) is scaled by a, with respect to (1).

Proof. The conditions of the lemma implies that there is some function $\beta \in \mathcal{K} \mathcal{L}$ such that

$$
\left|x\left(t, t_{0}, x_{0}\right)\right| \leq \beta\left(\left|x_{0}\right|, t-t_{0}\right), \quad \forall t \geq t_{0},
$$

for any $x_{0} \in \mathbb{R}^{n}$ and any $t_{0} \in \mathbb{R}$. By consideration above, for any $x_{0} \in \mathbb{R}^{n}$ and $a>0, z\left(t, t_{0}, x_{0}\right)=x\left(a t, a t_{0}, x_{0}\right)$ is the corresponding solution of (4), then

$$
\begin{aligned}
\left|z\left(t, t_{0}, x_{0}\right)\right| & =\left|x\left(a t, a t_{0}, x_{0}\right)\right|, \\
& \leq \beta\left(\left|x_{0}\right|, a\left[t-t_{0}\right]\right),
\end{aligned}
$$

for all $t \geq t_{0}$.

Consequently, for a homogeneous system (1), it may be obtained an extension of Proposition 2.

Proposition 3. Let $x\left(t, t_{0}, x_{0}\right)$ be a solution of the $r$ homogeneous system (1) with the degree $d$, for an initial condition $x_{0} \in \mathbb{R}^{n}$, and $t_{0} \in \mathbb{R}$. For any $\lambda>0$ and $a>0$, the system

$$
\frac{d w(t)}{d t}=a f(a \omega t, w(t)), t \geq t_{0}, t_{0} \in \mathbb{R},
$$

with $\omega=\lambda^{d}$, has a solution $w\left(t, t_{0}, w_{0}\right)=$ $\Lambda_{r}(\lambda) x\left(a \lambda^{d} t, a \lambda^{d} t_{0}, x_{0}\right)$, for all $t \geq t_{0}$, with the initial condition $w_{0}=\Lambda_{r}(\lambda) x_{0}$.

Proof. The proof repeats the arguments of Proposition 2.

Therefore, for $a=\lambda^{-d}$, the systems (1) and (5) have the same rates of convergence, and their corresponding solutions differ in amplitudes only.

\section{Finite-time Stability}

Assume that the system (1) is $r$-homogeneous with degree $d<0$ and GUAS (according to Lemma 1, the system (3) is also GUAS, for any $\omega>0$ in this case), assume also that $0<\mathcal{T}<+\infty$ where

$$
\begin{gathered}
\mathcal{T}=\sup _{\omega \in[0,1], x_{0} \in \mathbb{S}_{r}} T_{x_{0}}^{\omega}, \\
T_{x_{0}}^{\omega}=\sup _{t_{0} \in \mathbb{R}} \arg \inf _{\tau \geq 0}\left\{\left|x_{\omega}\left(t_{0}+\tau, t_{0}, x_{0}\right)\right|_{r} \leq 0.5\left|x_{0}\right|_{r}\right\},
\end{gathered}
$$

in other words, for all $\omega \in[0,1]$ and all initial conditions $x_{0} \in \mathbb{S}_{r}$ the time of convergence to the ball $B_{0.5}$ is less than $\mathcal{T}$, which is finite and independent in $t_{0} \in \mathbb{R}$ (the independence in $t_{0}$ follows from uniformity of stability). By Proposition 2, for any $x_{0} \in \mathbb{R}^{n}$ and $\lambda>0$, the solution of the system (3), for $\omega=\lambda^{d}$, has the form $x_{\omega}\left(t, t_{0}, x_{0}\right)=\Lambda_{r}(\lambda) x\left(\lambda^{d} t, \lambda^{d} t_{0}, \Lambda_{r}^{-1}(\lambda) x_{0}\right)$, for all $t \geq t_{0}$, with the initial conditions $x_{0}$. Thus $x\left(t, t_{0}, x_{0}\right)=$ $\Lambda_{r}^{-1}(\lambda) x_{\lambda^{d}}\left(\lambda^{-d} t, \lambda^{-d} t_{0}, \Lambda_{r}(\lambda) x_{0}\right)$, for any $x_{0} \in \mathbb{R}^{n}$, any $\lambda>0$, and all $t \geq t_{0}$. Next, for any $x_{0} \in \mathbb{S}_{r}$ and the corresponding trajectory $x\left(t, t_{0}, x_{0}\right)$ of the system (1), it is possible to define a sequence $x_{k}, k=1,2, \ldots$, such that $x_{k}=x\left(t_{k}, t_{0}, x_{0}\right)$, for some $t_{k}$, such that $\left|x_{k}\right|_{r}=2^{-k}$ (this sequence is well defined since the origin is attractive for (1)). Let us try to evaluate the upper estimate of the difference between $t_{k}-t_{k-1}$, for all $k=1,2, \ldots$, then obviously $t_{1}-t_{0} \leq \mathcal{T}$, by definition. Next, $x_{2}=x\left(t_{2}-t_{1}, t_{1}, x_{1}\right)$, and for $\lambda=2$ and some $\tilde{x}_{0} \in \mathbb{S}_{r}$, such that $\tilde{x}_{0}=\Lambda_{r}(2) x_{1}$, it is obtained

$$
\begin{aligned}
x\left(t_{2}-t_{1}, t_{1}, x_{1}\right)= & \Lambda_{r}^{-1}(\lambda) \\
& \times x_{\lambda^{d}}\left(\lambda^{-d}\left(t_{2}-t_{1}\right), \lambda^{-d} t_{1}, \Lambda_{r}(\lambda) x_{1}\right), \\
= & \Lambda_{r}^{-1}(2) x_{2^{d}}\left(2^{-d}\left(t_{2}-t_{1}\right), 2^{-d} t_{1}, \tilde{x}_{0}\right) .
\end{aligned}
$$

Since in (3), the value $\omega=2^{d}<1$, for $d<0$, if $2^{-d}\left(t_{2}-\right.$ $\left.t_{1}\right) \geq \mathcal{T}$, then $\left|x_{2^{d}}\left(2^{-d}\left(t_{2}-t_{1}\right), 2^{-d} t_{1}, \tilde{x}_{0}\right)\right|_{r} \leq 0.5\left|\tilde{x}_{0}\right|_{r}=$ 0.5 , and $\left|x\left(t_{2}-t_{1}, t_{1}, x_{1}\right)\right|_{r} \leq 0.25=\left|x_{2}\right|_{r}$. Therefore, $t_{2}-$ $t_{1} \leq 2^{d} \mathcal{T}$, and repeating these arguments, for all $k=1,2, \ldots$, it is obtained that $t_{k}-t_{k-1} \leq 2^{(k-1) d} \mathcal{T}$. Finally, the time of convergence of the trajectory $x\left(t, t_{0}, x_{0}\right)$ to the origin $T_{1, x_{0}}$ is

$$
\begin{aligned}
T_{1, x_{0}}=\sum_{k=1}^{+\infty} t_{k}-t_{k-1} & \leq \sum_{k=1}^{+\infty} 2^{(k-1) d} \mathcal{T}, \\
& =\frac{\mathcal{T}}{2^{d}} \sum_{k=1}^{+\infty} 2^{k d}, \\
& \leq \frac{\mathcal{T}}{2^{d}} \frac{1}{1-2^{d}},
\end{aligned}
$$

and the system is finite-time convergent from $\mathbb{S}_{r}$. Since for any initial conditions $x_{0} \in \mathbb{R}^{n}$, the corresponding time of convergence to the origin $T_{x_{0}}$ is the sum of $T_{1, x_{0}}$ and $T_{x_{0}}^{1}$, where $T_{x_{0}}^{1}$ is the time of convergence to $\mathbb{S}_{r}$ from the given initial conditions $x_{0}$, and $T_{x_{0}}^{1}$ is a function of initial conditions due to the global uniform asymptotic stability of (1), then the system (1) is globally uniformly finite-time convergent. Since system (1) is stable, it is GUFTS. Thus, the following result has been proven.

Theorem 1. Let (1) be r-homogeneous with degree $d<0$ and GUAS at the origin, assume also that $0<\mathcal{T}<+\infty$. Then, the system (1) is GUFTS at the origin.

The obtained result has a rather restrictive condition on the finiteness of $\mathcal{T}$ over all $\omega \in[0,1]$, but at least it provides a hint on restrictions under which the finite-time stability phenomenon is possible in time-varying systems. In the following, an academic example is introduced in order to show that the rate of convergence can be improved increasing the frequency for the finite-time case.

Consider the following nonlinear time-varying system

$$
\begin{aligned}
& \dot{x}_{1}(t)=x_{2}(t), \\
& \dot{x}_{2}(t)=-k_{1}\left\lceil x_{1}(t)\right\rfloor^{\frac{\gamma}{2-\gamma}}-k_{2} \Gamma(\omega t)\left\lceil x_{2}(t)\right\rfloor^{\gamma},
\end{aligned}
$$

where $\lceil\cdot\rfloor^{\gamma} \doteq|\cdot|^{\gamma} \operatorname{sign}(\cdot)$, with $\gamma \in(0,1), \Gamma(\omega t)=$ $\left(1+\sin ^{2} \omega t\right)$, and $k_{1}, k_{2}>0$ positive constants. The system (6)-(7) can be transformed into the following differential 


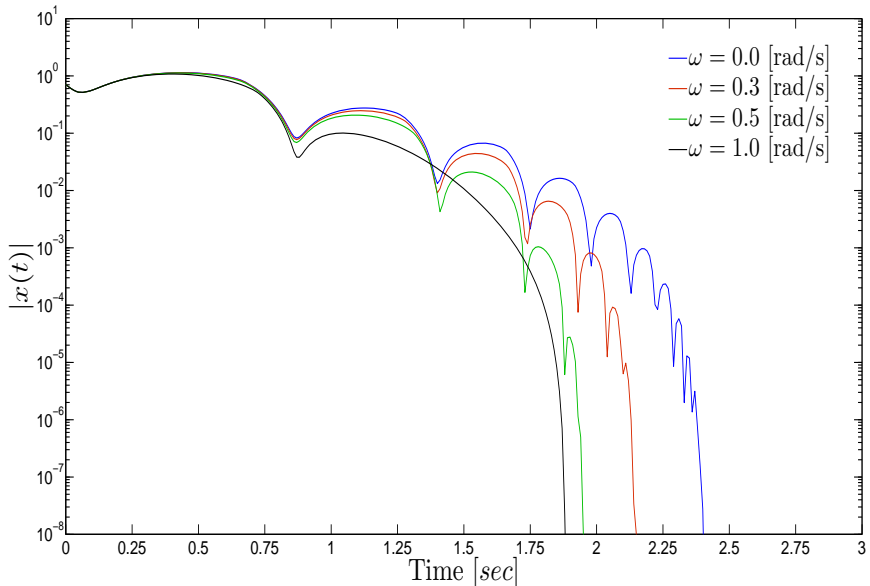

Figure 1. Finite-Time Convergence for Nonlinear Time-Varying System.

inclusion [28]

$$
\begin{aligned}
& \dot{x}_{1}(t) \in x_{2}(t), \\
& \dot{x}_{2}(t) \in-k_{1}\left\lceil x_{1}(t)\right\rfloor^{\frac{\gamma}{2-\gamma}}-[1,2] k_{2}\left\lceil x_{2}(t)\right\rfloor^{\gamma},
\end{aligned}
$$

System (8)-(9) is $r$-homogeneous with degree $d=\gamma-1<0$ for $\left(r_{1}, r_{2}\right)=(2-\gamma, 1)$, from the differential inclusion point of view given by [29]. Then, it is possible to demonstrate that such a system is GUFTS. Consider the following function

$$
V\left(x_{1}(t), x_{2}(t)\right)=\frac{2-\gamma}{2}\left|x_{1}(t)\right|^{\frac{2}{2-\gamma}}+\frac{1}{2 k_{1}}\left|x_{2}(t)\right|^{2} .
$$

Such a function $V\left(x_{1}(t), x_{2}(t)\right)$ is positive definite, radially unbounded, and continuously differentiable with $\dot{V}\left(x_{1}(t), x_{2}(\tau)\right) \leq-2 \frac{k_{2}}{k_{1}}\left|x_{2}(t)\right|^{\gamma+1}=-W\left(x_{1}(t), x_{2}(t)\right)$, negative semi-definite. Note that the limit $W\left(x_{1}(t), x_{2}(t)\right) \rightarrow$ 0 implies that $x_{2}(t)$ approaches to the set $E=$ $\left\{\left(x_{1}, x_{2}\right) \in \mathbb{R}^{2} \mid W\left(x_{1}(t), x_{2}(t)\right)=0\right\}$ as $t \rightarrow \infty$. Therefore, $W\left(x_{1}(t), x_{2}(t)\right)=0$ implies that $x_{2}(t)=0$ and thus $x_{1}(t)=$ cte. However, from (9), it is given that $\dot{x}_{2}(t) \in$ $-k_{1}\left\lceil x_{1}(t)\right\rfloor^{\frac{\gamma}{2-\gamma}}$, which implies also that $x_{1}(t)=0$. Thus, the set $E$ only contains the trivial solution, and GAUS is concluded [30]. Finally, from statements given by Corollary 4.3 in [29] and the equivalence of trajectories [28] between system (6)-(7) and (8)-(9), it is obtained that all the statements given by Theorem 1 are satisfied. Therefore, system (6)-(7) is GUFTS.

With the purpose of showing that the rate of convergence can be also improved increasing the frequency; some simulations for $\omega \in[0,1]$ have been done in MATLAB Simulink environment, with Euler discretization method and sampling time equal to $0.01[\mathrm{sec}]$. The simulation results, confirming previously given statements, are presented in Fig. 1. It is easy to see that increasing the frequency an improvement in the rate of convergence is obtained without the need to increase the gains $k_{1}, k_{2}$.

\section{ApPliCATION TO ADAPTIVE ESTIMATION}

In this section the previously proposed results will be applied to analyze the convergence of the error dynamics given by a nonlinear estimation algorithm.

\section{A. Problem statement}

Consider the following time-varying system:

$$
\frac{d x(t)}{d t}=\Gamma^{T}(t) \theta
$$

where $\Gamma: \mathbb{R} \rightarrow \mathbb{R}^{m}$ is a continuous function of time, and $\theta \in \mathbb{R}^{m}$. The regressor vector $\Gamma(t)$ is known and bounded, i.e. $\|\Gamma(t)\| \leq \Gamma_{+}$, satisfying the well-known persistence of excitation condition [19], and $\theta$ is the unknown parameter vector. It is assumed that $x(t)$ is available for measurements, and in order to estimate the vector $\theta$ the following nonlinear algorithm can be introduced

$$
\begin{aligned}
& \dot{\hat{x}}(t)=-k_{1}\lceil\hat{x}(t)-x(t)\rfloor^{\gamma}+\Gamma^{T}(t) \hat{\theta}(t), \quad \hat{x}(0)=0, \\
& \dot{\hat{\theta}}(t)=-k_{2}\lceil\hat{x}(t)-x(t)\rfloor^{2 \gamma-1} \Gamma(t), \quad \hat{\theta}(0)=[0 \ldots 0]^{T},
\end{aligned}
$$

where $k_{1}, k_{2}>0$ are positive gains, and the parameter $\gamma \in$ $(0.5,1)$ (for the case $\gamma=1$ the estimator reduces to the well known linear adaptive observer [19]). Let us define the errors $\tilde{x}(t)=\hat{x}(t)-x(t)$ and $\tilde{\theta}(t)=\hat{\theta}(t)-\theta$. Hence, the error dynamics is given by

$$
\begin{aligned}
& \dot{\tilde{x}}(t)=-k_{1}\lceil\tilde{x}(t)\rfloor^{\gamma}+\Gamma^{T}(t) \tilde{\theta}(t), \\
& \dot{\tilde{\theta}}(t)=-k_{2}\lceil\tilde{x}(t)\rfloor^{2 \gamma-1} \Gamma(t) .
\end{aligned}
$$

It is clear that the system (10)-(11) has the form (1), and it is also possible to express it in the form (3)

$$
\begin{aligned}
& \dot{\tilde{x}}(t)=-k_{1}\lceil\tilde{x}(t)\rfloor^{\gamma}+\Gamma^{T}(\omega t) \tilde{\theta}(t), \\
& \dot{\tilde{\theta}}(t)=-k_{2}\lceil\tilde{x}(t)\rfloor^{2 \gamma-1} \Gamma(\omega t) .
\end{aligned}
$$

System (10)-(11) is $r$-homogeneous with degree $d=\gamma-1$ for $\left(r_{1}, r_{2}, \ldots, r_{m+1}\right)=(1, \gamma, \ldots, \gamma)$. Note that $d<0$ for all $\gamma \in(0.5,1)$. It is possible to demonstrate that the system (10)-(11) is GUAS. Firstly, note that it is possible to rewrite system (10)-(11) as follows:

$$
\dot{\xi}(t)=A(t) \xi(t)+f(t, \tilde{x}(t))
$$

where $\xi(t)=\left[\begin{array}{ll}\tilde{x}(t) & \tilde{\theta}(t)^{T}\end{array}\right]^{T} \in \mathbb{R}^{1+m}, A(t)$ is a bounded and continuous matrix, for almost all $t \geq 0$, that is given by

$$
A(t)=\left[\begin{array}{cc}
-k_{1} & \Gamma^{T}(t) \\
-k_{2} \Gamma(t) & 0
\end{array}\right],
$$

and the nonlinear term $f: \rightarrow \mathbb{R}^{2} \rightarrow \mathbb{R}^{1+m}$ is given as follows:

$$
f(t, \tilde{x}(t))=\left[\begin{array}{c}
-k_{1}\left(\lceil\tilde{x}(t)\rfloor^{\gamma}-\tilde{x}(t)\right) \\
-k_{2}\left(\lceil\tilde{x}(t)\rfloor^{2 \gamma-1}-\tilde{x}(t)\right) \Gamma(t)
\end{array}\right] .
$$

Define

$$
W_{2}(t, \xi(t))=\xi^{T}(t) P(t) \xi(t),
$$

where $P(t)$ is a continuously differentiable, symmetric, bounded, and positive definite matrix, i.e. $0<c_{1} I \leq P(t) \leq$ $c_{2} I, \quad \forall t \geq 0$, which satisfies the following matrix differential equation:

$$
\dot{P}(t)=-P(t) A(t)-A^{T}(t) P(t)-Q(t), \quad \forall t \geq 0,
$$


where $Q(t) \geq c_{3} I>0, \quad \forall t \geq 0$, is a continuous, symmetric, and positive definite matrix. The existence of $P(t)$ and $Q(t)$ follows from the persistence of excitation condition of $\Gamma(t)$ (see, for example, [18], [31], [32]). Define also

$$
W_{1}(\tilde{x}(t), \tilde{\theta}(t))=\frac{\varsigma_{1}}{p} V_{1}^{p}(\tilde{x}(t), \tilde{\theta}(t))+\frac{\varsigma_{2}}{q} V_{1}^{q}(\tilde{x}(t), \tilde{\theta}(t)),
$$

with

$$
\begin{gathered}
V_{1}(\tilde{x}(t), \tilde{\theta}(t))=\frac{1}{2 \gamma}|\tilde{x}(t)|^{2 \gamma}+\frac{1}{2 k_{2}} \tilde{\theta}(t)^{T} \tilde{\theta}(t), \\
\varsigma_{1}=2 k_{1} \mu c_{2}(2 \gamma)^{p-1}, \varsigma_{2}=\frac{2 \mu c_{2}\left(k_{1}^{2}+k_{2}^{2} \Gamma_{+}^{2}\right)(2 \gamma)^{q-1}}{k_{1}},
\end{gathered}
$$

for some positive constants $p, q, \mu$. Introduce the following Lyapunov function candidate:

$$
W(t, \tilde{x}(t), \tilde{\theta}(t))=W_{1}(\tilde{x}(t), \tilde{\theta}(t))+W_{2}(t, \xi(t)) .
$$

Its derivative along the trajectories of the system (10)-(11) is given by

$$
\begin{aligned}
& \dot{W}(t, \tilde{x}(t), \tilde{\theta}(t))=\left(\varsigma_{1} V_{1}^{p-1}(\tilde{x}(t), \tilde{\theta}(t))\right. \\
&\left.+\varsigma_{2} V_{1}^{q-1}(\tilde{x}(t), \tilde{\theta}(t))\right) \dot{V}_{1}(\tilde{x}(t), \tilde{\theta}(t)) \\
&+\xi^{T}(t)\left[\dot{P}(t)+P(t) A(t)+A^{T}(t) P(t)\right] \xi(t) \\
&+2 \xi^{T}(t) P(t) f(\tilde{x}(t), t) .
\end{aligned}
$$

Using the fact that $\dot{V}_{1}(\tilde{x}(t), \tilde{\theta}(t))=-k_{1}|\tilde{x}(t)|^{3 \gamma-1}$, $2 X^{T} P Y \leq \frac{1}{\mu} X^{T} P X+\mu Y^{T} P Y$ for any $Y \in \mathbb{R}^{1+m}$ and $\mu>0$, it follows that

$$
\begin{gathered}
\dot{W}(t, \tilde{x}(t), \tilde{\theta}(t)) \leq-k_{1}\left(\varsigma_{1} V_{1}^{p-1}(\tilde{x}(t), \tilde{\theta}(t))+\right. \\
\left.\varsigma_{2} V_{1}^{q-1}(\tilde{x}(t), \tilde{\theta}(t))\right)\left(|\tilde{x}(t)|^{3 \gamma-1}\right)- \\
\xi^{T}(t) Q(t) \xi(t)+\frac{1}{\mu} \xi^{T}(t) P(t) \xi(t)+\mu f^{T}(\tilde{x}(t), t) P(t) f(\tilde{x}(t), t),
\end{gathered}
$$

$$
\dot{W}(t, \tilde{x}(t), \tilde{\theta}(t)) \leq-\eta\|\xi\|^{2}+\mu c_{2}\|f\|^{2}
$$$$
-k_{1}\left(\varsigma_{1} V_{1}^{p-1}(\tilde{x}(t), \tilde{\theta}(t))+\varsigma_{2} V_{1}^{q-1}(\tilde{x}(t), \tilde{\theta}(t))\right)|\tilde{x}(t)|^{3 \gamma-1} \text {, }
$$

where $\eta=c_{3}-\frac{c_{2}}{\mu}$. Note that $V_{1}(\tilde{x}(t), \tilde{\theta}(t)) \geq \frac{1}{2 \gamma}|\tilde{x}(t)|^{2 \gamma}$, and the term $\mu c_{2}\|f\|^{2}$ can be bounded as follows:

$$
\begin{aligned}
\mu c_{2}\|f\|^{2} \leq \mu c_{2}\left(2 k_{1}^{2}(\right. & \left.|\tilde{x}(t)|^{2 \gamma}+|\tilde{x}(t)|^{2}\right)+ \\
& \left.+k_{2}^{2} \Gamma_{+}^{2}\left(|\tilde{x}(t)|^{4 \gamma-2}+|\tilde{x}(t)|^{2}\right)\right),
\end{aligned}
$$

therefore

$$
\begin{aligned}
& \dot{W}(t, \tilde{x}(t), \tilde{\theta}(t)) \leq-\eta\|\xi\|^{2} \\
& +2 \mu c_{2} k_{1}^{2}|\tilde{x}(t)|^{2 \gamma}-\frac{k_{1} \varsigma_{1}}{(2 \gamma)^{p-1}}|\tilde{x}(t)|^{\gamma(2 p+1)-1} \\
& +2 \mu c_{2} k_{1}^{2}|\tilde{x}(t)|^{2}-\frac{k_{1} \varsigma_{2}}{(2 \gamma)^{q-1}}|\tilde{x}(t)|^{\gamma(2 q+1)-1} \\
& +\mu c_{2} k_{2}^{2} \Gamma_{+}^{2}\left(|\tilde{x}(t)|^{4 \gamma-2}+|\tilde{x}(t)|^{2}\right) .
\end{aligned}
$$

Then, for all $|\tilde{x}(t)|>1$, it is given that $|\tilde{x}(t)|^{4 \gamma-2}+$ $|\tilde{x}(t)|^{2} \leq 2|\tilde{x}(t)|^{2}$, and

$$
\begin{aligned}
& \dot{W}(t, \tilde{x}(t), \tilde{\theta}(t)) \leq-\eta\|\xi\|^{2} \\
& +2 \mu c_{2} k_{1}^{2}|\tilde{x}(t)|^{2 \gamma}-\frac{k_{1} \varsigma_{1}}{(2 \gamma)^{p-1}}|\tilde{x}(t)|^{\gamma(2 p+1)-1} \\
& +2 \mu c_{2}\left(k_{1}^{2}+k_{2}^{2} \Gamma_{+}^{2}\right)|\tilde{x}(t)|^{2}-\frac{k_{1} \varsigma_{2}}{(2 \gamma)^{q-1}}|\tilde{x}(t)|^{\gamma(2 q+1)-1} .
\end{aligned}
$$

Thus, the parameters $p$ and $q$ will be designed such that every positive term, in $\dot{W}(t, \tilde{x}(t), \tilde{\theta}(t))$, is compensated, i.e. such that

$$
\begin{aligned}
|\tilde{x}(t)|^{2 \gamma} & =|\tilde{x}(t)|^{\gamma(2 p+1)-1}, \\
|\tilde{x}(t)|^{2} & =|\tilde{x}(t)|^{\gamma(2 q+1)-1} .
\end{aligned}
$$

Therefore, in order to ensure that $\dot{W}(t, \tilde{x}(t), \tilde{\theta}(t)) \leq$ $-\eta\|\xi\|^{2}$, for all $|\tilde{x}(t)|>1$, the following constraints are obtained

$$
p=\frac{\gamma+1}{2 \gamma}, \quad q=\frac{3-\gamma}{2 \gamma}
$$

Note that when $\gamma \in(0.5,1)$, it is obtained that $p \in(1,1.5)$ and $q \in(1,2.5)$. Thus, $W(t, \tilde{x}(t), \tilde{\theta}(t))$ is positive definite, radially unbounded, continuous in $\mathbb{R}^{1+m}$ and continuously differentiable for all $|\tilde{x}(t)|>1$. For the case $|\tilde{x}(t)| \leq 1$, it is given that $|\tilde{x}(t)|^{4 \gamma-2}+|\tilde{x}(t)|^{2} \leq 2$, and

$$
\begin{aligned}
& \dot{W}(t, \tilde{x}(t), \tilde{\theta}(t)) \leq-\eta\|\xi\|^{2}+2 \mu c_{2} k_{2}^{2} \Gamma_{+}^{2} \\
& +2 \mu c_{2} k_{1}^{2}|\tilde{x}(t)|^{2 \gamma}-\frac{k_{1} \varsigma_{1}}{(2 \gamma)^{p-1}}|\tilde{x}(t)|^{\gamma(2 p+1)-1} \\
& \quad+2 \mu c_{2} k_{1}^{2}|\tilde{x}(t)|^{2}-\frac{k_{1} \varsigma_{2}}{(2 \gamma)^{q-1}}|\tilde{x}(t)|^{\gamma(2 q+1)-1}
\end{aligned}
$$

Since the positive terms $|\tilde{x}(t)|^{2 \gamma}$ and $|\tilde{x}(t)|^{2}$, can be compensated by the negative terms $|\tilde{x}(t)|^{\gamma(2 p+1)-1}$ and $|\tilde{x}(t)|^{\gamma(2 q+1)-1}$, for all $\tilde{x}$; it is obtained that $\forall|\tilde{x}(t)| \leq 1$

$$
\dot{W}(t, \tilde{x}(t), \tilde{\theta}(t)) \leq-\eta\|\xi\|^{2}+2 \mu c_{2} k_{2}^{2} \Gamma_{+}^{2} .
$$

It is possible to demonstrate that $W(\tilde{x}(t), \tilde{\theta}(t)) \geq$ $\frac{\varsigma_{1}}{p} \alpha^{p}\|\xi\|^{2 p}+\frac{\varsigma_{2}}{q} \alpha^{q}\|\xi\|^{2 q}+c_{1}\|\xi\|^{2}$, with $\alpha=\min \left(\frac{1}{2 \gamma}, \frac{1}{2 k_{2}}\right)$; and since $p \in(1,1.5)$ and $q \in(1,2.5)$, it is given that $W(\tilde{x}(t), \tilde{\theta}(t)) \geq \phi\|\xi\|^{5}, \forall|\tilde{x}(t)| \leq 1$, with $\phi \geq \frac{\varsigma_{1}}{p} \alpha^{p}+\frac{\varsigma_{2}}{q} \alpha^{q}+$ $c_{1}$. Therefore, it follows that $\forall|\tilde{x}(t)| \leq 1$

$$
\dot{W}(t, \tilde{x}(t), \tilde{\theta}(t)) \leq-\frac{\eta}{\phi^{\frac{2}{5}}} W^{\frac{2}{5}}(\tilde{x}(t), \tilde{\theta}(t))+2 \mu c_{2} k_{2}^{2} \Gamma_{+}^{2},
$$

and US is obtained. Thus, based on the statements given by Corollary 5 , the following result has been proven.

Theorem 2. Let the vector $\Gamma(t)$ be persistently exciting. Then, the system (10)-(11) is GUAS at the origin.

Since system (10)-(11) is also homogeneous, the rate of convergence for its modified version (12)-(13) can be evaluated using Lemma 1 from the convergence of the original system (10)-(11). The modification (12)-(13) corresponds to a frequency change in the regressor vector, that is a usual instrument in the adaptive estimation. According to the results of Proposition 2 and Lemma 1, for the linear estimator with 
$\gamma=1$ the speed of error convergence should not be modified, while for the nonlinear observer (12)-(13) with $\gamma \in(0.5,1)$ the frequency $\omega$ may impact the convergence (note that the form of the function $\beta \in \mathcal{K} \mathcal{L}$, from Lemma 1, is unknown, in general a rescaling by $\omega^{-1 / d}$ of the initial conditions may cancel the rate improvement by $\omega t$ ).

\section{B. Example}

Consider the Pendulum-Cart system depicted in Fig. 2. A

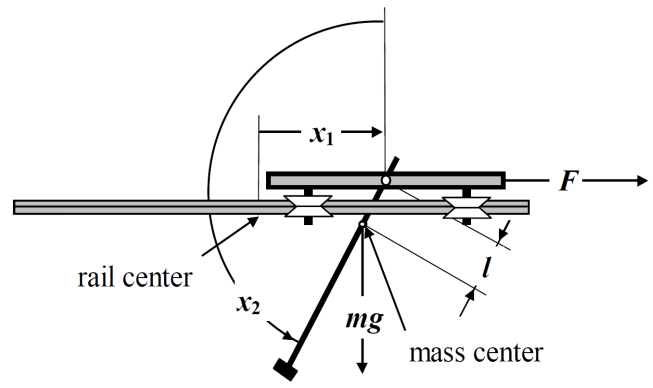

Figure 2. Pendulum-Cart System.

pendulum rotates in a vertical plane around an axis located on a cart. The cart can move along a horizontal rail, lying in the plane of rotation. The system state is a vector $x=$ $\left[x_{1}, x_{2}, x_{3}, x_{4}\right]^{T}$, where $x_{1}$ is the cart position, $x_{2}$ is the angle between the upward direction and the pendulum, measured counterclockwise $\left(x_{2}=0\right.$ for the upright position of the pendulum), $x_{3}$ is the cart velocity, and $x_{4}$ is the pendulum angular velocity. A control force $F$, parallel to the rail, is applied to the cart. It is produced by a DC flat motor controlled by a pulsewidth-modulation (PWM) voltage signal $u$, and $F=p_{1} u$. The system control $u$ takes values in the interval $[-0.5,0.5]$. The total mass of the pendulum and cart is denoted by $m$. The distance from the axis of rotation of the pendulum to the center of mass of the system is $l$. The moment of inertia of the pendulum with respect to its axis on the cart is represented by $J_{p}$. The cart friction is compound of two forces: the static and the viscous friction proportional to the cart velocity, $f_{c} x_{3}$ and $f_{s} \operatorname{sign}\left(x_{3}\right)$, respectively. There is also a friction torque in the angular motion of the pendulum, proportional to the angular velocity, $f_{p} x_{4}$. The system dynamics is described by the following equations

$$
\begin{aligned}
\dot{x}_{1}(t) & =x_{3}(t), \\
\dot{x}_{2}(t) & =x_{4}(t), \\
\dot{x}_{3}(t) & =\frac{a_{1} w_{1}(x(t), u(t))+a_{2}\left(x_{2}(t)\right) w_{2}(x(t))}{d\left(x_{2}(t)\right)}, \\
\dot{x}_{4}(t) & =\frac{a_{2}\left(x_{2}(t)\right) w_{1}(x(t), u(t))+w_{2}(x(t))}{d\left(x_{2}(t)\right)}, \\
y(t) & =\left[x_{1}(t) x_{2}(t)\right]^{T},
\end{aligned}
$$

where

$$
\begin{aligned}
& m=m_{c}+m_{p}, a_{1}=l^{2}+\frac{J}{m}, a_{2}\left(x_{2}(t)\right)=l \cos \left(x_{2}(t)\right), \\
& w_{1}(x(t), u(t))=p_{1} u(t)-f_{s} \operatorname{sign}\left(x_{3}(t)\right)-f_{c} x_{3}(t) \\
& \quad-m l x_{4}^{2}(t) \sin \left(x_{2}(t)\right), \\
& w_{2}(x(t))=m l g \sin \left(x_{2}(t)\right),-f_{p} x_{4}(t), \\
& d\left(x_{2}(t)\right)=J+m l^{2} \sin ^{2}\left(x_{2}(t)\right) .
\end{aligned}
$$

The parameters of the model are given in Table I. Let us

Table I

Parameters of the Pendulum-Cart System.

\begin{tabular}{|c|c|c|}
\hline Name & Description & Unit \\
\hline \hline$m_{c}$ & Car mass & $0.5723[\mathrm{~kg}]$ \\
\hline$m_{p}$ & Pendulum mass & $0.12[\mathrm{~kg}]$ \\
\hline$l$ & Distance from the rotation axis to the center of mass & $0.0196[\mathrm{~m}]$ \\
\hline$f_{C}$ & Dynamic friction coefficient & $0.5[\mathrm{Ns} / \mathrm{m}]$ \\
\hline$f_{S}$ & Dry friction coefficient & $1.1976[\mathrm{~kg} / \mathrm{m}]$ \\
\hline$f_{p}$ & Rotational friction coefficient & $0.1354[\mathrm{Nms} / \mathrm{rad}]$ \\
\hline$J$ & Moment of inertia of the pendulum & $0.0039\left[\mathrm{kgm} \mathrm{m}^{2}\right]$ \\
\hline$g$ & Gravity & $9.81\left[\mathrm{~m} / \mathrm{s}^{2}\right]$ \\
\hline$p_{1}$ & Control force to PWM signal ratio & $9.4[\mathrm{~N}]$ \\
\hline
\end{tabular}

assume that it is necessary to identify the friction parameters, i.e. $f_{s}, f_{c}, f_{p}$, and the control force to PWM signal radio, i.e. $p_{1}$, therefore, the dynamics given by (17) can be rewritten as follows

$$
\dot{x}_{3}(t)=\Gamma^{T}(t) \theta(t)+f(x(t)),
$$

where

$\Gamma^{T}(t)=\frac{a_{1}}{d\left(x_{2}(t)\right)}\left[\begin{array}{llll}-\operatorname{sign}\left(x_{3}(t)\right) & -x_{3}(t) & -x_{4}(t) & u(t)\end{array}\right]$,

$\theta^{T}(t)=\left[\begin{array}{llll}f_{s} & f_{c} & f_{p} & p_{1}\end{array}\right]$,

$u(t)=0.4 \sin (\omega t)+0.1 \cos (0.1 \omega t)$,

$f(x(t))=\frac{a_{2}\left(x_{2}(t)\right) m l g \sin \left(x_{2}(t)\right)-a_{1} m l x_{4}^{2}(t) \sin \left(x_{2}(t)\right)}{d\left(x_{2}(t)\right)}$.

Note that it is enough to consider dynamics (20) in order to identify the corresponding parameters. Assume that the state vector $x(t)$ is measurable. The parameter identification algorithm takes the following form

$$
\begin{aligned}
\dot{\hat{x}}_{3}(t) & =-k_{1}\left\lceil\hat{x}_{3}(t)-x_{3}(t)\right\rfloor^{\gamma}+\Gamma^{T}(t) \hat{\theta}(t)+f(x(t)), \\
\dot{\hat{\theta}}(t) & =-k_{2}\left\lceil\hat{x}_{3}(t)-x_{3}(t)\right\rfloor^{2 \gamma-1} \Gamma(t),
\end{aligned}
$$

where $\hat{x}_{3}(0)=0$ and $\hat{\theta}(0)=\left[\begin{array}{llll}0.8 & 0.1 & 0 & 4.5\end{array}\right]^{T}$. Let us consider the case $\gamma=0.70$ with $k_{1}=35, k_{2}=3.5$. Some simulations, for different values of $\omega$, have been done in MATLAB Simulink environment, with Euler discretization method and sampling time equal to $0.01[\mathrm{sec}]$. The simulation results, confirming Lemma 1 statements, are presented in Figs. 3-4 in comparison with the linear estimation algorithm for $\gamma=1$. As a conclusion, increasing the frequency, it is possible to improve the rate of convergence for the nonlinear algorithm in certain limits, while for the linear algorithm there is no significant improvement.

Therefore, application of the nonlinear homogeneous algorithms may serve for improvement of the rate of estimation 


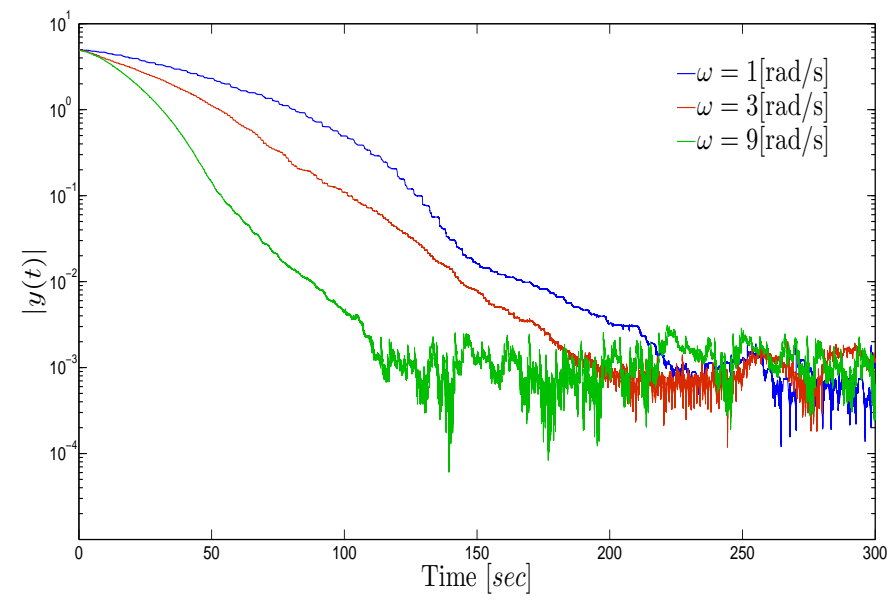

Figure 3. Estimation Error Convergence for Nonlinear Adaptive Observer $(\gamma=0.70), \xi(t)=\left[\begin{array}{cc}\tilde{x}_{3}(t) & \tilde{\theta}^{T}(t)\end{array}\right]^{T}$.

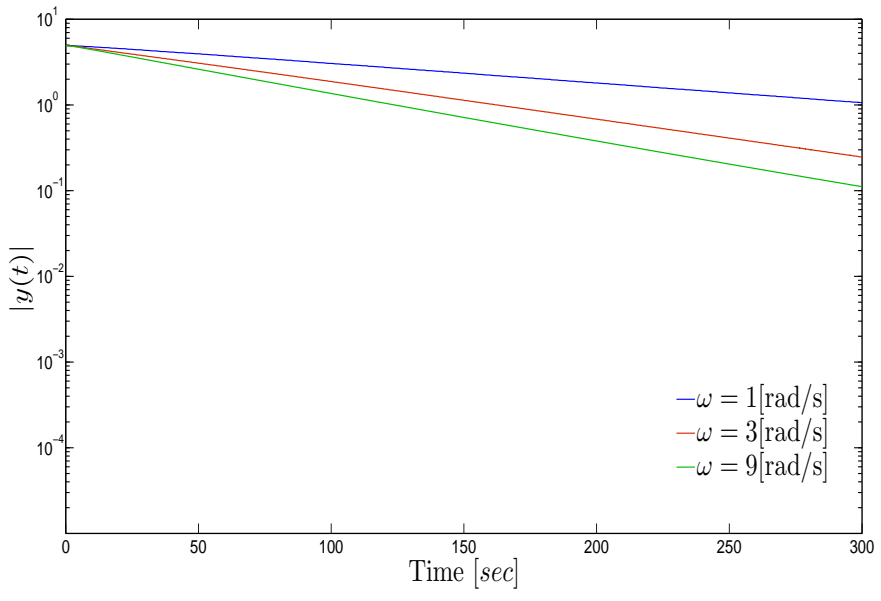

Figure 4. Estimation Error Convergence for Linear Adaptive Observer $(\gamma=$ $1), \xi(t)=\left[\begin{array}{cc}\tilde{x}_{3}(t) & \tilde{\theta}^{T}(t)\end{array}\right]^{T}$.

depending on the available excitation frequency of the timevarying regressor.

\section{CONCLUSIONS}

The homogeneity theory extensions are obtained for timevarying systems. It is shown that for any degree of homogeneity the solutions of a homogeneous system are interrelated subject to the time rescaling. Next, this fact is utilized in order to show that local uniform asymptotic stability of homogeneous systems implies global one, and that for nonlinear homogeneous systems with non-zero degree global asymptotic stability for a parameter endorses this property for an arbitrary value of this parameter. The possibility of finite-time stability in time-varying systems is discussed. Efficiency of the proposed approach is demonstrated for an adaptive estimation problem benchmark. Application of the developed results for analysis and design of control or estimation algorithms in timevarying systems is a direction of future research. Improvement of rate of convergence in the adaptive estimation algorithm by parameter tuning based on the proposed technique is another possible direction of future work.

\section{ACKNOWLEDGMENT}

The authors gratefully acknowledge the financial support from CONACyT(209731)-CNRS(222629) Bilateral Cooperation Project Mexico-France, and Fondo de Colaboración de Facultad de Ingeniería e Instituto de Ingeniería de la UNAM IGSBAS-122-2014. This work was also supported in part by the Government of Russian Federation (Grant 074-U01) and the Ministry of Education and Science of Russian Federation (Project 14.Z50.31.0031).

\section{REFERENCES}

[1] A. Bacciotti and L. Rosier, Liapunov Functions and Stability in Control Theory, vol. 267 of Lecture Notes in Control and Inform. Sci. Berlin: Springer, 2001.

[2] V. Andrieu, L. Praly, and A. Astolfi, "Homogeneous approximation, recursive observer design, and output feedback," SIAM J. Control Optimization, vol. 47, no. 4, pp. 1814-1850, 2008.

[3] H. Hermes, Differential Equations: Stability and Control, vol. 109 of Lecture Notes in Pure Appl. Math., ch. Homogeneous coordinates and continuous asymptotically stabilizing feedback controls, pp. 249-260. Marcel Dekker, 1991.

[4] Y. Hong, "Finite-time stabilization and stabilizability of a class of controllable systems," Systems\&Control Lett., vol. 46, pp. 231-236, 2002.

[5] L. Rosier, "Homogeneous Lyapunov function for homogeneous continuous vector field," Systems\&Control Lett., vol. 19, pp. 467-473, 1992.

[6] H. Hermes, "Nilpotent and high-order approximations of vector field systems," SIAM Review, vol. 33, no. 2, pp. 238-264, 1991.

[7] T. Ménard, E. Moulay, and W. Perruquetti, "Homogeneous approximations and local observer design," ESAIM: Control, Optimization and Calculus of Variations, vol. 19, no. 3, pp. 906-929, 2013.

[8] A. Levant, "Homogeneity approach to high-order sliding mode design," Automatica, vol. 41, no. 5, pp. 823-830, 2005.

[9] S. Bhat and D. Bernstein, "Geometric homogeneity with applications to finite-time stability," Mathematics of Control, Signals and Systems, vol. 17, pp. 101-127, 2005.

[10] L. Grüne, "Homogeneous state feedback stabilization of homogeneous systems," SIAM J. Control Optimization, vol. 38, no. 4, pp. 1288-1314, 2000.

[11] M. Kawski, Homogeneous feedback stabilization, vol. 7 of Progress in systems and control theory: New trends in systems theory. Birkhäuser, 1991.

[12] E. Moulay and W. Perruquetti, "Finite time stability and stabilization of a class of continuous systems," J. Mathematical Analysis Applications, vol. 323, no. 2, pp. 1430-1443, 2006.

[13] R. Sepulchre and D. Aeyels, "Stabilizability does not imply homogeneous stabilizability for controllable systems," SIAM J. Control Optimization, vol. 34, no. 5, pp. 1798-1813, 1996.

[14] V. Zubov, "On systems of ordinary differential equations with generalized homogenous right-hand sides," Izvestia vuzov. Mathematica., vol. 1, pp. 80-88, 1958. in Russian.

[15] D. Efimov and W. Perruquetti, "Oscillations conditions in homogenous systems," in Proc. NOLCOS'10, (Bologna), pp. 1379-1384, 2010.

[16] E. Bernuau, A. Polyakov, D. Efimov, and W. Perruquetti, "On iss and iiss properties of homogeneous systems," in Proc. European Control Conference (ECC) 2013, (Zürich), 2013.

[17] E. Bernuau, A. Polyakov, D. Efimov, and W. Perruquetti, "Verification of iss, iiss and ioss properties applying weighted homogeneity," Systems \& Control Letters, vol. 62, no. 12, pp. 1159-1167, 2013.

[18] P. A. Ioannou and J. Sun, Robust Adaptive Control. New Jersey, USA: Prentice Hall, Inc., 1996.

[19] K. Narendra and A. Annaswamy, Stable Adaptive Systems. Mineola, NY: Dover Publications, 2005.

[20] J. Peuteman and D. Aeyels, "Averaging results and the study of uniform asymptotic stability of homogeneous differential equations that are not fast time-varying," SIAM J. Control Optimization, vol. 37, no. 4, pp. 997-1010, 1999.

[21] Y. Orlov, "Finite time stability and robust control synthesis of uncertain switched systems," SIAM J. Control Optimization, vol. 43, no. 4, pp. 1253-1271, 2005.

[22] E. Roxin, "On finite stability in control systems," Rendiconti del Circolo Matematico di Palermo, vol. 15, pp. 273-283, 1966. 
[23] P. Dorato, "An overview of finite-time stability," in Current Trends in Nonlinear Systems and Control (L. Menini, L. Zaccarian, and C. T. Abdallah, eds.), Systems \& Control: Foundations \& Applications, pp. 185194, Boston: Birkhäuser, 2006.

[24] S. Nersesov, W. Haddad, and Q. Hui, "Finite-time stabilization of nonlinear dynamical systems via control vector lyapunov functions," Journal of the Franklin Institute, vol. 345, pp. 819-837, 2008.

[25] A. Polyakov, "Nonlinear feedback design for fixed-time stabilization of linear control systems," IEEE Transactions on Automatic Control, vol. 57, no. 8, pp. 2106-2110, 2012.

[26] E. Cruz-Zavala, J. Moreno, and L. Fridman, "Uniform robust exact differentiator," IEEE Transactions on Automatic Control, vol. 56, no. 11, pp. 2727-2733, 2011.

[27] H. Khalil, Nonlinear Systems. Upper Saddle River, New Jersey: Prentice Hall, 3rd ed., 2002.

[28] A. Filippov, Differential Equations with Discontinuous Righthand Sides. Dordrecht, The Netherlands: Kluwer Academic Publishers, 1988.

[29] W. P. Emmanuel Bernuau, Denis Efimov and A. Polyakov, "On an extension of homogeneity notion for differential inclusions," in 2013 European Control Conference, (Zürich, Switzerland), pp. 2204-2209, 2013.

[30] W. Desch, H. Logemann, E. P. Ryan, and E. D. Sontag, "Meagre functions and asymptotic behaviour of dynamical systems," Nonlinear Analysis: Theory, Methods \& Applications, vol. 44, no. 8, pp. 10871109, 2001.

[31] G. Besançon, Nonlinear Observers and Applications, vol. 363 of Lecture Notes in Control and Information Science. Springer-Verlang Berlin Heidelberg, 2007.

[32] D. Efimov, C. Edwards, and A. Zolghadri, "A note on improvement of adaptive observer robustness," in Proccedings of the 19th IFAC World Congress, (Cape Town, South Africa), pp. 558-562, 2014.

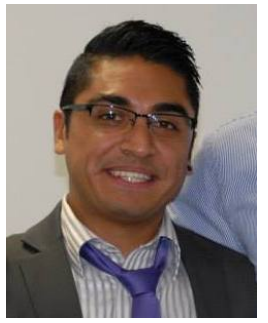

Héctor Ríos was born in México D.F. in 1984. He received the B.Sc degree in Electric and Electronic Engineering, M.Sc and Ph.D degrees in Electric Engineering, Automatic Control, from "Universidad Nacional Autónoma de México" (UNAM) in 2008 2010 and 2014, respectively. From 2014 to 2015 he was working in the Non-A team at Inria Lille center. His professional activities have been concentrated in observation of linear and nonlinear systems, observation of switched systems, fault detection, isolation and identification problems, high-order sliding-mode control and its applications. He has published more than 20 publications including journal articles, book chapters and conferences articles.

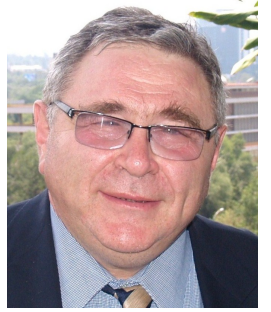

Leonid M. Fridman received an M.S. degree in mathematics from Kuibyshev (Samara) State University, Samara, Russia, in 1976, a Ph.D. degree in applied mathematics from the Institute of Control Science, Moscow, Russia, in 1988, and a Dr. Sc. degree in control science from Moscow State University of Mathematics and Electronics, Moscow, Russia, in 1998. From 1976 to 1999 , he was with the Department of Mathematics, Samara State Architecture and Civil Engineering University. From 2000 to 2002, he was with the Department of Postgraduate Study and Investigations at the Chihuahua Institute of Technology, Chihuahua, Mexico. In 2002, he joined the Department of Control Engineering and Robotics, Division of Electrical Engineering of Engineering Faculty at National Autonomous University of Mexico (UNAM), Mexico. His research interests are Variable Structure Systems. He is currently a Chair of TC on Variable Structure Systems and Sliding mode control of IEEE Control Systems Society, Associated Editor of the Journal of Franklin Institute, and Nonlinear Analysis: Hybrid Systems, and the Conference Editorial Board of IEEE Control Systems Society. He is an author and editor of seven books and 15 special issues devoted to the sliding mode control. He is a winner of Scopus prize for the best cited Mexican Scientists in Mathematics and Engineering 2010. He was working as an invited professor in 20 universities and research laboratories of Argentina, Australia, Austria, France, China, Germany, Italy, Israel, and Spain.

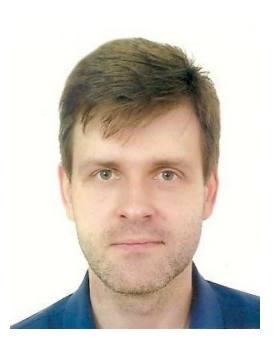

Denis Efimov received the Ph.D. degree in Automatic Control from the Saint-Petersburg State Electrical Engineering University (Russia) in 2001, and the Dr.Sc. degree in Automatic control in 2006 from Institute for Problems of Mechanical Engineering RAS (Saint-Petersburg, Russia). From 2000 to 2009 he was research fellow of the Institute for Problems of Mechanical Engineering RAS, Control of Complex Systems Laboratory. From 2006 to 2011 he was working in the LSS (Supelec, France), the Montefiore Institute (University of Liege, Belgium) and the Automatic control group at IMS lab (University of Bordeaux I, France). Since 2011 he joined the Non-A team at Inria Lille center. He is a member of several IFAC TCs and a Senior member of IEEE. His main research interests include nonlinear oscillation analysis, observation and control, switched and nonlinear system stability.

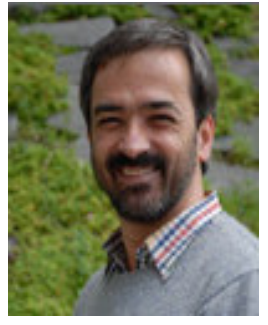

Jaime A. Moreno was born in Colombia and he received his $\mathrm{PhD}$ degree (Summa cum Laude) in Electrical Engineering (Automatic Control) from the Helmut-Schmidt University in Hamburg, Germany in 1995. The Diplom-Degree in Electrical Engineering (Automatic Control) from the Universität zu Karlsruhe, Karlsruhe, Germany in 1990, and the Licentiate-Degree (with honors) in Electronic Engineering from the Universidad Pontificia Bolivariana, Medellin, Colombia in 1987. He is full Professor of Automatic Control and the Head of the Electrical and Computing Department at the Institute of Engineering from the National University of Mexico (UNAM), in Mexico City. He is member of the Technical Board of IFAC. He is the author and editor of 8 books, 4 book chapters, 1 patent, and author and co-author of more than 300 papers in refereed journals and conference proceedings. His current research interests include robust and non-linear control with application to biochemical processes (wastewater treatment processes), the design of nonlinear observers and higher order sliding mode control. 


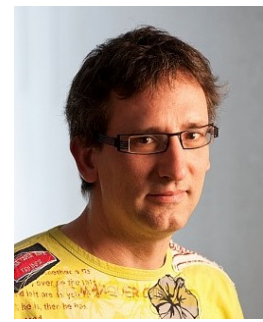

Wilfrid Perruquetti was born in 1968 in Saint Gilles, France. In 1991, he received an M.Sc. in Automatic Control and graduated from "Institut Industriel du Nord" (French "Grande Ecole"). In 1994, he obtained a Ph.D. in Automatic Control, then joined the "Ecole Centrale de Lille" (French "Grande Ecole") as an Assistant Professor in 1995, where he is actually Full Professor (since 2003). He belongs to CRIStAL (CNRS) and to the Non-A project (Inria Lille-Nord Europe). He is vice-deputy of INS2I CNRS, permanent head of Non-A Inria, and was from (2010 to 2014) scientific project manager at ANR, from (2007 to 2009) a representative of the French Ministry of Education and Research (DGRI). He is member of several councils and of several societies (IFAC, TC 1.3, 2.3, 2.5 (past) and 9.2 (Chair), SEE member). He has published more than 170 journals, book chapters and conference papers (more than 60 journal papers) and is co-editor with Jean-Pierre Barbot of the books "Sliding Mode Control in Engineering", Marcel Dekker and "Chaos in Automatic control", CRC Taylor \& Francis. He is currently working on stability analysis (including various kinds of stability concepts), stabilization (in particular finite-time stabilization), sliding mode control of nonlinear and delay systems, observation of state variables and identification of system parameters. His main fields of application concern robotics, in particular mobile robots (path planning, stabilization, coordination...), robotic manipulators (trajectory generation and control) and electrical actuators (DC motor, induction motor, stepper motor...). 